\title{
Nonlinear analysis of the heartbeats in public patient ECGs using an automated PD2i algorithm for risk stratification of arrhythmic death
}

\author{
James E Skinner \\ Jerry MAnchin \\ Daniel N Weiss \\ Vicor Technologies, Inc., Bangor, PA, \\ USA
}

Correspondence: James E Skinner

Vicor Technologies, Inc., 399 Autumn

Drive, Bangor, PA I80 I3, USA

Tel +l 5708975797

Email jskinner@vicortech.com

\begin{abstract}
Heart rate variability (HRV) reflects both cardiac autonomic function and risk of arrhythmic death (AD). Reduced indices of HRV based on linear stochastic models are independent risk factors for AD in post-myocardial infarct cohorts. Indices based on nonlinear deterministic models have a significantly higher sensitivity and specificity for predicting AD in retrospective data. A need exists for nonlinear analytic software easily used by a medical technician. In the current study, an automated nonlinear algorithm, the time-dependent point correlation dimension (PD2i), was evaluated. The electrocardiogram (ECG) data were provided through an National Institutes of Health-sponsored internet archive (PhysioBank) and consisted of all 22 malignant arrhythmia ECG files (VF/VT) and 22 randomly selected arrhythmia files as the controls. The results were blindly calculated by automated software (Vicor 2.0, Vicor Technologies, Inc., Boca Raton, FL) and showed all analyzable VF/VT files had PD2i $<1.4$ and all analyzable controls had PD2i $>1.4$. Five VF/VT and six controls were excluded because surrogate testing showed the RR-intervals to contain noise, possibly resulting from the low digitization rate of the ECGs. The sensitivity was $100 \%$, specificity $85 \%$, relative risk $>100$; $p<0.01$, power $>90 \%$. Thus, automated heartbeat analysis by the time-dependent nonlinear $\mathrm{PD} 2 \mathrm{i}$-algorithm can accurately stratify risk of $\mathrm{AD}$ in public data made available for competitive testing of algorithms.
\end{abstract}

Keywords: heart rate variability, sudden death, ventricular arrhythmias, nonlinear, chaos

Brain and behavior have been shown in animal studies to be important variables in the initiation of ventricular fibrillation (VF) and its consequence, arrhythmic death (AD) (Skinner and Reed 1981; Skinner and Verrier 1982; Skinner et al 1983). The analysis of heart rate variability (HRV), which noninvasively provides real-time trending of beat-to-beat changes in the clinical ECG, reflects covariations in autonomic function and later occurrences of VF (Akselrod et al 1981; Stein et al 1994). Decreased $\mathrm{HRV}$ indices are frequently found among patients with myocardial infarction (MI), congestive heart failure (CHF), diabetes, and alcoholic cardiomyopathy (Kleiger et al 1987; Stein et al 1994). The indices are independent risk factors for mortality in the post-MI and advanced CHF patients (Kleiger et al 1987; Coumel et al 1991; Stein et al 1994; Ho et al 1997). HRV indices based on brief observational windows (10- to 15-min) have the same predictability for VF and AD as those based on 24-hr records (Fei et al 1996).

The various HRV indices have not been widely adopted because no single measurement has yet demonstrated sufficient statistical predictability of AD in individuals, ie, they have inadequate sensitivity and specificity to be of any clinical utility. The limited performance may be related to assumptions of the model underlying the algorithm 
used to measure the beat-to-beat variation. For example, an algorithm such as SDNN, which is based on a linear stochastic model, presumes the data variation is random, is distributed around a mean, and has had removed all arrhythmias, movement artifacts and heart rate changes caused by uncontrolled nonstationary events. We know, however, that heartbeat intervals have brief internal correlations, are not random, and thus violate the model assumptions for most algorithms. In contrast, an algorithm based on a nonlinear deterministic model presumes that the variation is caused physiologically and has no beat-to-beat errors. Thus, the nonlinear measures should be inherently more accurate because the data fit the underlying models, and apparently this is the case (Ho et al 1997; Skinner et al 1998).

The consideration about the fundamental nature of the cardiac rhythm and its measurement has stimulated the development of new HRV analyses (Wagner and Persson, 1998), but has left the clinical utility an open issue. Comparative tests among algorithms in retrospective data suggest that the time-dependent nonlinear algorithms show the greatest promise for clinical HRV analyses (Braun et al 1998; Skinner et al 1998; Marwan et al 2002).

Our objective was to evaluate the ability of a nonlinear algorithm that is completely automated (Vicor 2.0, Vicor Technologies, Inc., Boca Raton, FL) and easy for a medical technician to apply to standard ECGs containing arrhythmias, artifacts, and other nonstationarities. This algorithm, the point correlation dimension (PD2i), was selected because of its insensitivity to nonstationarities in ECG data (Skinner et al 1993) and its superior performance compared with other linear and nonlinear algorithms in the prediction of documented lethal arrhythmogenesis (Skinner et al 1998). We used digitized ECGs posted in a public database, PhysioBank (Goldberger et al 2000). These ECGs have been made available for competitive testing of HRV algorithms. All 22 files from patients with malignant arrhythmias (ventricular fibrillation [VF] or sustained ventricular tachycardia [VT]) were selected and, as the controls, 22 randomly selected files with arrhythmias (common premature atria and ventricular contractions, and less common junctional, supraventricular and ventricular complexes).

\section{Methods}

\section{The clinical data}

The clinical data were from the MIT-BIH (Harvard's Beth Israel Hospital ECG Archive) contributions to the National Institutes of Health (NIH)-sponsored PhysioBank
(Goldberger et al 2000), and included all of the malignant ventricular arrhythmia ECGs $(n=22)$, and a random selection of the arrhythmia ECGs $(n=22)$ to serve as the controls. Half of the controls were randomly selected from the arrhythmia archive of over 4,000 ECGs (filenames 100-124) with common arrhythmia types (premature atrial and ventricular beats) and the other half from the arrhythmia archive containing less common, but challenging arrhythmias (filenames 200-234). The latter included; those with complex ventricular, junctional, and supraventricular arrhythmias that were thought to present significant difficulty for algorithmic processing. Documentation of the arrhythmia rates and types are provided in the PhysioBank, along with some relevant clinical data.

All ECGs were digitized at either 250-Hz (malignant ventricular arrhythmias) or 360-Hz (arrhythmias) and were approximately 20 to 30 minutes in length. Either VF or VT could be seen in the malignant arrhythmia ECGs, except in a few cases where documentation occurred within a few seconds to minutes after the ECG file ended. There were 25 men and 32 women in the arrhythmia archive; the gender for the malignant ventricular arrhythmia archive was unknown. The malignant arrhythmia ECGs were collected during the period from 1975 to 1979.

\section{Pd2i analyses}

All analyses were performed using completely blinded and automated procedures. The files were downloaded, renamed with a code name, and then sent to another site to be analyzed by a person without knowledge of their origin or outcome. The software (Vicor 2.0, Vicor Technologies, Inc.) ran in a completely automated fashion once the input ECG file name and its start and stop times were provided. Episodes of VF or VT were not included in the analyses. A common rule was used for determining the minimum number of data points (Ni) to resolve a given dimension (Kostelich and Swinney 1989). Successful time-dependent tracking of degrees of freedom (dimension) in ECG data with numerous arrhythmias and artifacts has been previously demonstrated for the PD2i algorithm (Skinner et al 1993). The same PD2i parameters were used in Vicor 2.0 as in previous publications $(\mathrm{Tau}=1, \mathrm{LC}=0.30, \mathrm{CC}=0.40, \mathrm{PL}=0.15, \mathrm{PI}=4$ ) (Skinner et al 1991, 1993, 1994, 1998, 2000; Vybiral and Skinner 1993; Elbert et al 1994; Skinner and Molnar 1999). Outliers greater than 2.8 standard deviations from the mean were removed from the relative risk-interval series by a linear interpolation spline, but only if PD2i scores less than 1.4 were associated with them in time; this was done because 
large amplitude arrhythmias or artifacts of constant size can achieve low-dimensional PD2i scores; otherwise artifacts and arrhythmias are generally rejected from analysis by the $\mathrm{LC}$ and $\mathrm{CC}$ criteria in the PD2i algorithm (Skinner et al 1993). The PD2 $i$ is insensitive to nonstationarities in the data (Skinner et al 1994), so no attempt was made to search for stationary sub-epochs upon which to perform the PD2i analyses.

\section{Noise analyses}

Relative risk (RR)-interval data contain descretization errors $(0.8 \%$ for $250-\mathrm{Hz}$ digitization of the ECG) and amplifier noise ( 2 microvolts, $0.5 \%$ ). They also contain RR-interval detection error compared with the visual standard; the 3-point running-window operator employed by Vicor 2.0 , is set at 60 msec between points and this template-matching is accurate within $\pm 1 \mathrm{msec}(0.1 \%$ error $)$. A small amount of noise in the $\mathrm{RR}$ data is tolerated by the PD2i algorithm (ie, \pm 5 integers; $1 \mathrm{mV}=1 \mathrm{int} ; 0.7 \%$ ) (Skinner et al 1994). So the excess noise produced by the low digitization rate of the $250-\mathrm{Hz}$ ECG may present a problem compared to previous studies in which $1000-\mathrm{Hz}$ digitization was employed (ie, $1000-\mathrm{Hz}$ digitization produces only one-fourth the descretization error of $250-\mathrm{Hz}$ ). Therefore each RR-interval file was first inspected for noise content by Vicor 2.0 to assure a valid interpretation of the nonlinear PD2i result. To assure that the data were not contaminated by noise, two tests were run, the more conventional Surrogate Test (Theiler et al 1992; Schreiber and Schmitz 1996 ) and the \%N Test used in our previous studies (Skinner et al 1991, 1993, 1994, 1998, 2000).

\section{Statistical analysis}

The sensitivity, specificity, and RR statistics assessed predictability, using Fisher's Exact Test to determine the significance of each true or false vs VF/VT or noVF/VT contingency (Prism Software, Irvine, CA). Powering of the study was determined $(>90 \%)$ using the proportions method of Fleiss (1981).

\section{Results}

\section{Clinical characteristics}

The study utilized 44 ECGs, each of which had a known outcome (22 VF/VT and 22 noVF/VT). The clinical data for each subject were not reported in the PhysioBank for the VF/VT subjects, but the peer-reviewed manuscript (Goldberger et al 2000) declares that the archived ECGs are valid open source data to be used in determining algorithmic quantifications, such as those employed in this study.

\section{Analytic results}

Figure 1 shows data and analytic results from a normal healthy individual (A) and from two typical ECGs in the database (B and $C$ ). Figure 2 shows accepted PD2i values for 7 Arrhythmia controls and 7 VF/VT subjects. The PD2i of the VF/VT subjects all fall below the 1.4 level (horizontal bar). This 1.4 cut-point was a priori adopted from our first published clinical study (Skinner et al 1993), as this criterion was based on receiver-operator curves to maximize sensitivity and specificity. The primary results shown in Figure 2 for the first half of the non-excluded subjects are virtually the same as those for the second half, as documented in Table 1.

\section{Clinical prediction of arrhythmic death}

Table 1 shows the results from all 44 subjects. The coded file names are in column 1 and the corresponding file names in the MIT-BIH database are in column 2. The column labeled PD2i Test indicates for each ECG file those with PD2 $\mathrm{i} \leq 1.4$ (POS), those with PD2i $>1.4$ (NEG) and those rejected (Rej) because of noise content (failed Surrogate Test, failed $\% \mathrm{~N}$ Test). The PD2i of the RR-intervals and the PD2i for the randomized-phase surrogates made from the RR intervals were not statistically significantly different for 11 ECGs (Surrogate Test, ns), and these cases were a priori exclusions from analysis. These same surrogate-excluded files showed $\% \mathrm{~N}$ to be less than $30 \%$, and were thus automatically rejected by the Vicor 2.0 software (PD2i Test, Rej). The rejected ECG files included all cases of atrial fibrillation and high arrhythmia rate (ie, $>10 \%$ of beats). The origin of the arrhythmias was of sinus, ventricular, or a mix of both. The VF/VT Outcomes (column 4) were expressed as true negative (TN), true positive (TP), false negative (FN), false positive (FP) or a priori excluded (Exc). Of the 33 nonrejected files, 17 were TP, 13 TN, 3 FP, and 0 FN. This resulted in a sensitivity $=100 \%$, specificity $=85 \%$, positive predictive value $=85 \%$, negative predictive value $=100 \%$, and relative risk $>100(\mathrm{p}<0.01$, Fisher Exact Test). These were all sufficiently powered tests $(>90 \%)$. The hypothesis that the VF/VT predictions were by chance alone was rejected ( $<<0.001$, Binomial Probability Test).

\section{Discussion}

\section{Interpretation of results}

The automated PD2i algorithm (Vicor 2.0) appears to work well on ECG data digitized at $250-\mathrm{Hz}$ instead of the more usual 1000-Hz employed in previous studies (Skinner et al 1993, 1994, 1998, 2000), but this lower digitization rate may contribute to the greater number of exclusions from analysis than previously observed (Skinner et al 1993) because of 

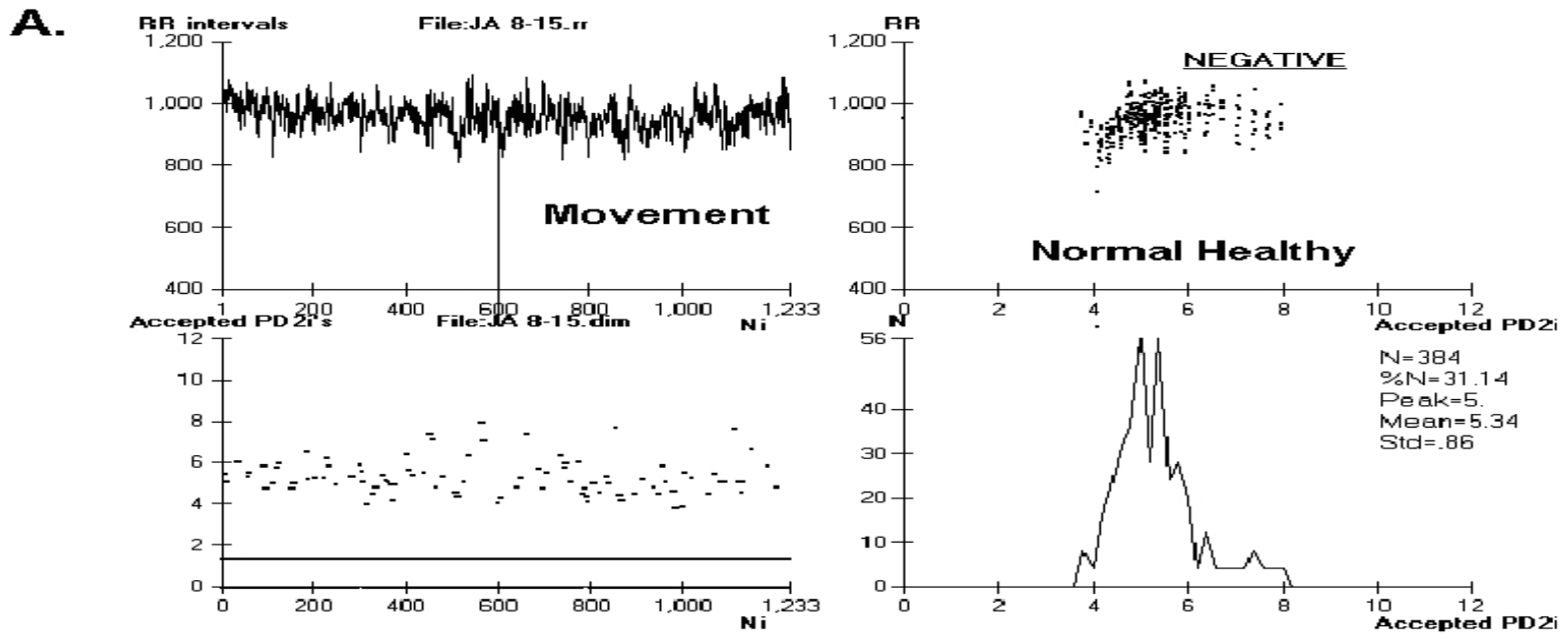

B.
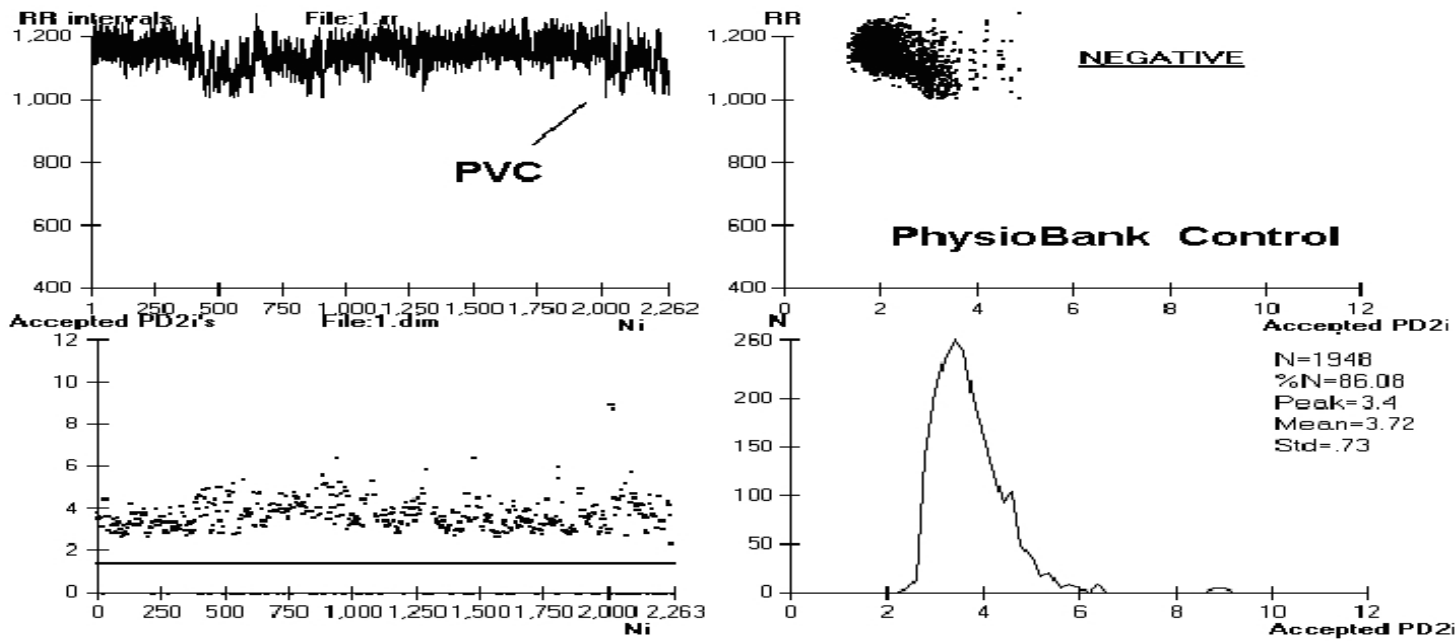

C.
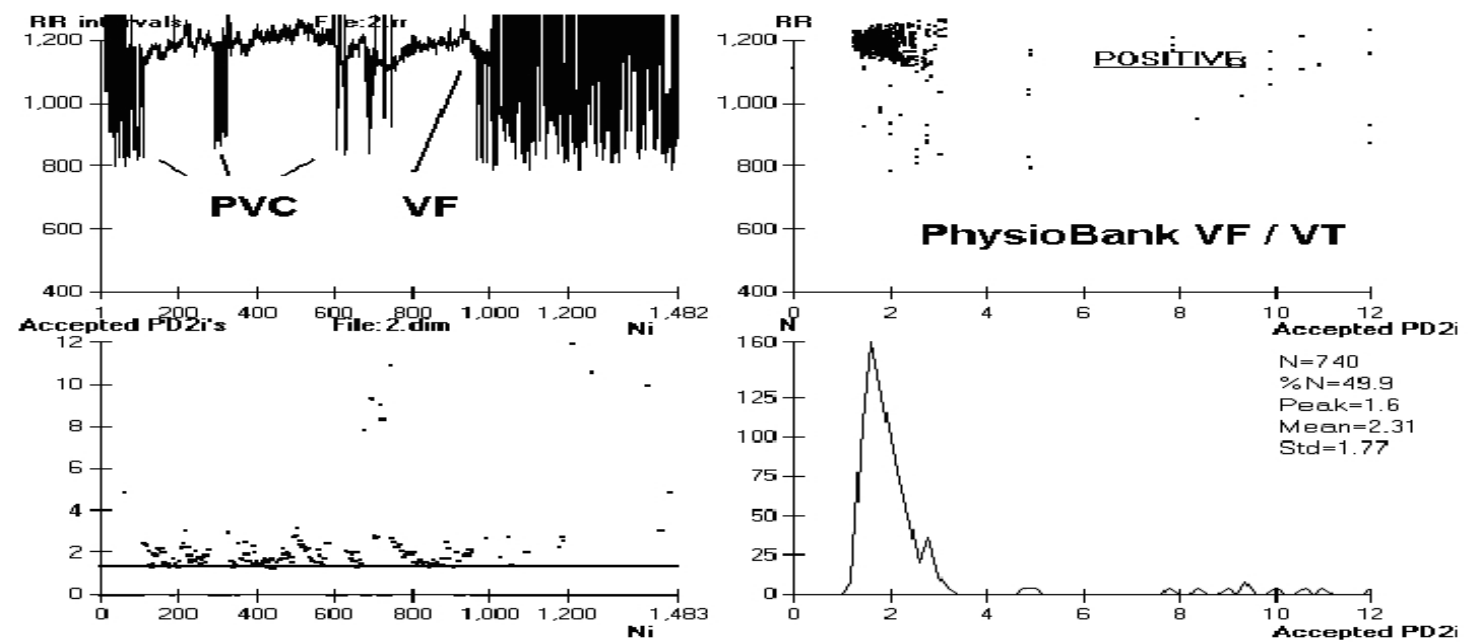

Figure I Relative risk (RR)-intervals and associated PD2i's determined from the electrocardiogram (ECG) of a normal healthy person and two types of PhysioBank patients. A. Shows RR data and corresponding PD2i results from a normal healthy subject ( 15 min ECG). B. Shows results from an arrhythmia control patient (30 min ECG, PhysioBank). C. Shows results from a VF/VT patient (30 min ECG, PhysioBank). PD2i values are expressed in degrees of freedom (dimensions) and RR values in msec; VF is included in the analysis for illustrative purposes only. Parts A-C each show 4 individual plots for comparing the data and the analytic results: upper left the RR-intervals (RRi), lower left the corresponding PD2i, upper right the joint plot of RRi and PD2i, lower right the histogram of the accepted PD2i values with its associated statistics, including \%N.The ECG for the RRi shown in Part C. manifests VF at 17 minutes from the start and the PD2i beforeVF shows repeated low-dimensional excursions fall below I.4 (line). The ECG shown in Part B. did not manifest any PD2i below 1.4 throughout the 30-min period. The test result indicated in the upper right plot (underlined) was completely automated by the Vicor 2.0 software once the input file was entered. 
Arrhythmia Controls
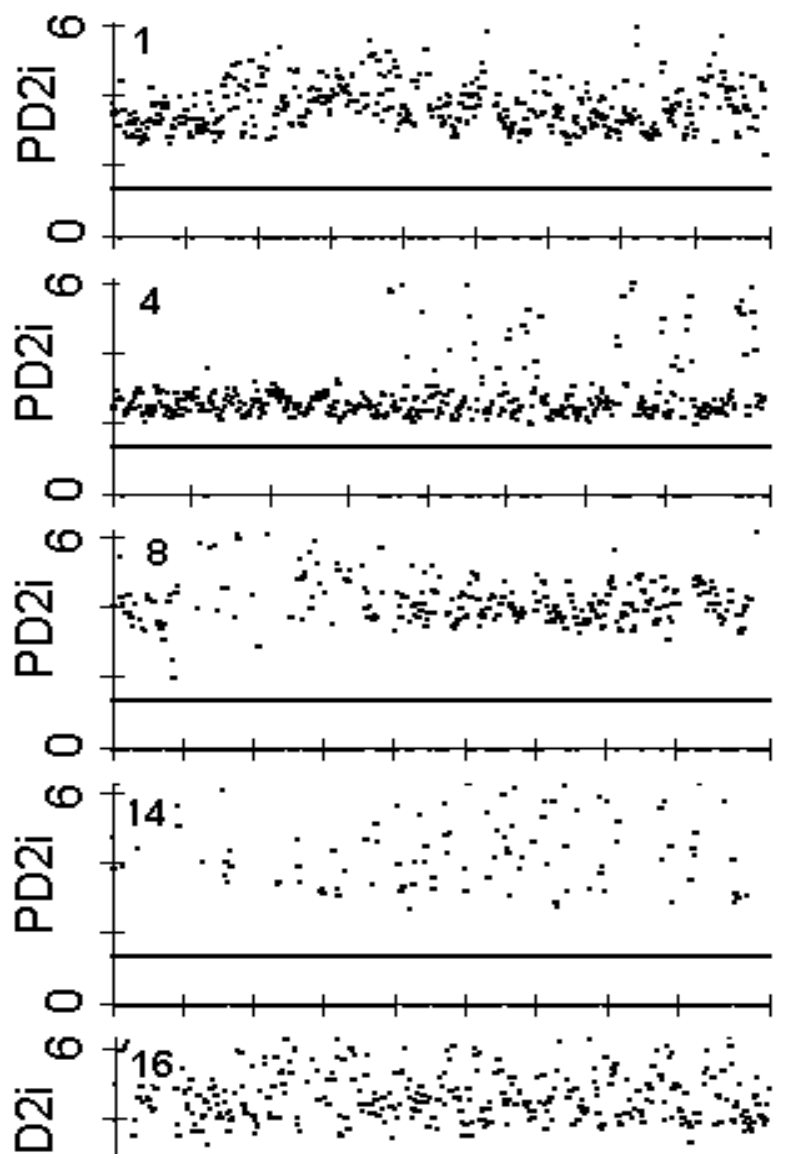

모

๑
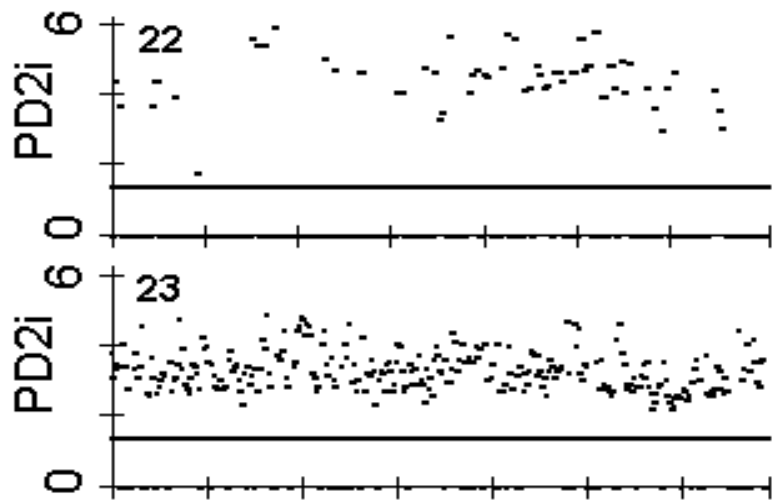

Heartbeats
VF / VT Patients (PhysioBank)
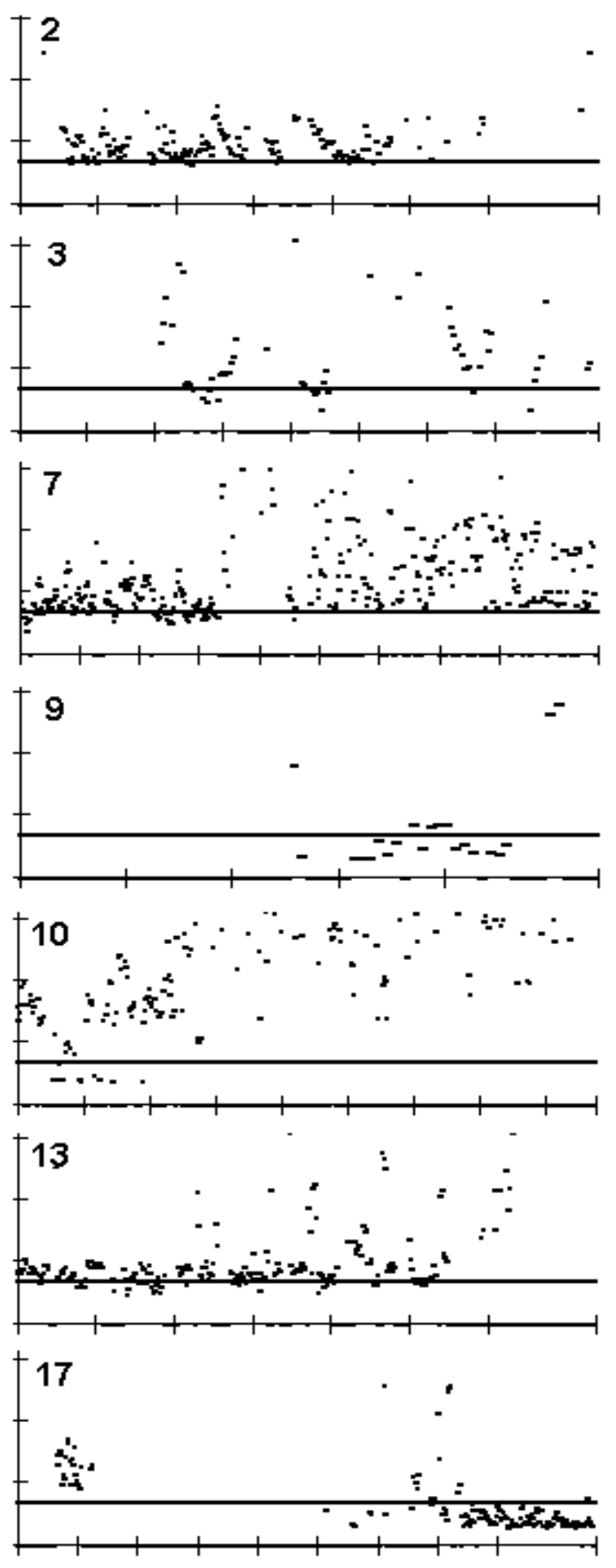

Heartbeats

Figure 2 PD2i traces from arrhythmia controls and malignant arrhythmia subjects for the first half of the data set. PD2i values are expressed in degrees of freedom (dimensions) and the heartbeats are those obtained in a 15- to 30-min ECG up to the end or point of VF/VT onset. Note the low-dimensional excursions of the PD2i in the VF/VT subjects. The horizontal a priori criterion lines are set at 1.4 degrees of freedom and completely separate the two groups of patients. The rejected PD2i points are due to noise produced by arrhythmias and movement artifacts, but \%N remains high enough in these patients for valid analysis. Surrogate- and \%N-rejected files $(\mathrm{n}=\mathrm{II}$ ) are not shown as they were a priori rejected (5VF/VT, 6 controls). These rejected files contained all subjects with atrial fibrillation and the highest arrhythmia rates (ie, greater than $10 \%$ arrhythmias in all beats). 
Table I PD2i of heartbeats predicts VF/VT in PhysioBank ECGs ${ }^{\mathbf{a}}$

\begin{tabular}{|c|c|c|c|c|c|}
\hline Coded file & MIT-BIH & PD2i test & VF/VT outcome* & Surrogate test & $\% \mathbf{N}$ test \\
\hline $\mathrm{I}$ & 100 & NEG & TN & $\mathrm{p}<0.01$ & 86 \\
\hline 2 & 422 & POS & TP & $\mathrm{p}<0.01$ & 50 \\
\hline 3 & 419 & POS & TP & $\mathrm{p}<0.01$ & 32 \\
\hline 4 & 107 & NEG & $\mathrm{TN}$ & $\mathrm{p}<0.01$ & 92 \\
\hline 5 & 200 & Rej & Exc & ns & 17 \\
\hline 6 & 424 & Rej & Exc & ns & 22 \\
\hline 7 & 611 & POS & TP & $\mathrm{P}<0.01$ & 78 \\
\hline 8 & 114 & NEG & $\mathrm{TN}$ & $\mathrm{p}<0.0 \mathrm{I}$ & 67 \\
\hline 9 & 430 & POS & TP & $\mathrm{P}<0.01$ & 31 \\
\hline 10 & 426 & POS & TP & $\mathrm{P}<0.01$ & 41 \\
\hline II & 119 & $\operatorname{Rej}$ & Exc & ns & 23 \\
\hline 12 & 602 & POS & TP & $\mathrm{p}<0.01$ & 33 \\
\hline 13 & 607 & POS & TP & $\mathrm{P}<0.01$ & 60 \\
\hline 14 & 228 & NEG & $\mathrm{TN}$ & $\mathrm{P}<0.0 \mathrm{I}$ & 37 \\
\hline 15 & 425 & Rej & Exc & ns & 23 \\
\hline 16 & 104 & NEG & $\mathrm{TN}$ & $\mathrm{p}<0.01$ & 68 \\
\hline 17 & 418 & POS & TP & $\mathrm{p}<0.01$ & 36 \\
\hline 18 & 428 & POS & TP & $\mathrm{p}<0.0 \mathrm{I}$ & 87 \\
\hline 19 & 101 & NEG & $\mathrm{TN}$ & $\mathrm{p}<0.0 \mathrm{I}$ & 64 \\
\hline 20 & 421 & POS & TP & $\mathrm{p}<0.01$ & 67 \\
\hline 21 & 420 & POS & TP & $\mathrm{p}<0.01$ & 76 \\
\hline 22 & 106 & NEG & $\mathrm{TN}$ & $\mathrm{P}<0.01$ & 33 \\
\hline 23 & 121 & NEG & $\mathrm{TN}$ & $\mathrm{p}<0.01$ & 81 \\
\hline 24 & 610 & POS & TP & $\mathrm{p}<0.01$ & 33 \\
\hline 25 & 605 & Rej & Exc & ns & 27 \\
\hline 26 & 231 & POS & FP & $\mathrm{p}<0.01$ & 54 \\
\hline 27 & 217 & NEG & $\mathrm{TN}$ & $\mathrm{P}<0.0 \mathrm{I}$ & 61 \\
\hline 28 & 201 & Rej & Exc & ns & 13 \\
\hline 29 & 423 & POS & TP & $\mathrm{P}<0.01$ & 33 \\
\hline 30 & 429 & POS & TP & $\mathrm{P}<0.01$ & 38 \\
\hline 31 & 113 & NEG & $\mathrm{TN}$ & $\mathrm{p}<0.01$ & 39 \\
\hline 32 & 116 & POS & FP & $\mathrm{P}<0.01$ & 92 \\
\hline 33 & 203 & Rej & Exc & Ns & 22 \\
\hline 34 & 124 & NEG & $\mathrm{TN}$ & $\mathrm{P}<0.0 \mathrm{I}$ & 52 \\
\hline 35 & 427 & POS & TP & $\mathrm{P}<0.01$ & 68 \\
\hline 36 & 612 & POS & TP & $\mathrm{p}<0.01$ & 46 \\
\hline 37 & 614 & Rej & Esc & ns & 23 \\
\hline 38 & 221 & Rej & Exc & ns & 11 \\
\hline 39 & 213 & Rej & Exc & ns & 3 \\
\hline 40 & 615 & Rej & Exc & ns & 17 \\
\hline $4 I$ & 223 & NEG & $\mathrm{TN}$ & $\mathrm{P}<0.0 \mathrm{I}$ & 70 \\
\hline 42 & 233 & POS & $\mathrm{FP}$ & $\mathrm{p}<0.01$ & 33 \\
\hline 43 & 609 & POS & TP & $\mathrm{p}<0.01$ & 45 \\
\hline 44 & 103 & NEG & $\mathrm{TN}$ & $\mathrm{p}<0.0 \mathrm{I}$ & 82 \\
\hline
\end{tabular}

Notes: Sensitivity = 100\%; specificity = 85\%; $<0.0$ (Fisher Exact); Coded File: coded file names, as shown in Figure 2, to cross reference with PhysioBank file names; MIT-BIH, PhysioBank file names for the ECG data (ie, from MIT and Beth Israel Hospital archives); PD2i Test, analytic result, where NEG (negative) indicates PD2i > I.4, POS (positive) indicates PD2i $\leq 1.4$, and Rej (rejection) indicates data rejected by the \%N criterion; all analytic results were automated by the Vicor 2.0 software; VF/VT Outcome, given the VF/VT or no VF/VT outcome by PhysioBank, the PD2i predicted a True Negative (TN), a True Positive (TP), a False Negative (FN; none indicated), or a False Positive (FP); Exc indicates the prediction could not be made because the Surrogate and \%N Tests together could not affirm that the data were noise-free; Surrogate Test, probability that the mean PD2i of the data and the mean PD2i of the randomized-phase surrogate were the same, as tested by a 2-tailed t-test; ns indicates they were not statistically significantly different, and therefore the data could not be presumed to be noise-free; \% N Test, \% of accepted PD2i values, where values less than 30 lead to rejection of the data by the Vicor 2.0 software for a valid PD2i analysis.

the higher descretization error. All of the other sources of noise (high arrhythmia rate, movement artifacts, amplifier noise) are additive with the descretization error and together determine the number of failed Surrogate Tests and $\% \mathrm{~N}$
Tests. The PD2i of the heartbeats in publicly archived ECGs is a statistically significant predictor of VF/VT, with there being no FN cases, and thus confirms previous observations in retrospective data that did not employ automated analyses 
(Skinner et al 1993, 1998). The comparison of results suggests that PD2 $\mathrm{i}$ analysis of the ECG by a technician using the Vicor 2.0 software is equal to that by an expert in nonlinear signal analysis doing all of the analytic operations by hand.

\section{What the PD2i measures}

There can be little doubt that cardiac vulnerability to lethal arrhythmogenesis is regulated by the brain and autonomic nervous system, for blockade of the descending efferent pathways, at the level of the frontal cortex (Skinner and Reed 1981) or amygdala (Carpeggiani et al 1992) or at the level of the peripheral cardiac nerves (Ebert et al 1970), will prevent VF in animals following coronary artery occlusion. These higher neural systems also participate in the regulation of the heart rate, just as do the afferent-efferent loops through the medulla that underlie the baroreflex and respiratory sinus arrhythmia and the loops through the hypothalamus that regulate blood $\mathrm{pH}$ and temperature.

During quiet wakefulness each of these multiple loops contributes to the nonlinear regulation of the RR-intervals by its interaction on its own time scale (Marwan et al 2002; Braun et al 1998). The degrees of freedom resulting from the net nonlinear interactions are approximately 6 , ranging in time from 4 to 8 (Figure 1A). This type of tied-together regulation results in the erratic RR-interval series that is clinically referred as the "normal sinus rhythm." It is the physiological evolution of abnormal dynamics in this tied-together, nonlinear regulation that leads to the type of altered rhythm that is associated with susceptibility to VF in hospitalized patients. Such a dynamic is associated with a reduction in the number of degrees of freedom, as shown in Figures 1 and 2. This reduction in the degrees of freedom in the heartbeats below 1.4 could result from greater "cooperation" or phase relationship among the independent regulators. We do not yet know how this or any alternative mechanisms may actually produce the changed dynamics, but we do know it is organized by the higher nervous system (Meyer et al 1996; Skinner 2007).

The PD2 $\mathrm{i}$ algorithm is a new measure of heartbeat regulation that, instead of being based on a linear stochastic model like the standard deviation and power spectra, is a nonlinear measure of variability not requiring any a priori presumptions about the data in its underlying model. The motivation for developing the PD2 $\mathrm{i}$ algorithm was the simple observation that the series of heartbeat intervals is not a sequence of independent numbers randomly distributed around a mean, as is required by any algorithm based on a linear stochastic model. An indepth discussion of these fundamental issues ("When the mean is meaningless"), along with the presentation of other novel nonlinear algorithms in the assessment of HRV, is provided elsewhere (Braun et al 1998; Stiedl and Meyer 2002, 2003). The poorer performance of the linear measures, we believe, results from the fundamental misconception that analyzing an inherently nonlinear and correlated time series by a linear stochastic technique is all right. On theoretical grounds this usage commits a serious type-II error.

The PD2i measures the degree of interaction, or "cooperation," among the competing neural loops that simultaneously control the dynamics of the cardiac rhythm and the vulnerability of the heart to lethal arrhythmogenesis. Accentuated cooperation or breakdown of the normal coupling among these competing autonomic nervous loops (ie, PD2i $\leq 1.4$ ) is somehow able to set the stage for the evolution of lethal arrhythmogenesis. The mechanism for the coordination of such "cooperation" is presently unknown, but there can be little doubt that it is organized within the brain and is projected onto the nodal and ventricular tissues of the heart through the autonomic nervous system. The heart transplant recipient manifests a resting heartbeat dynamics of PD2 $\mathrm{i}=1.0$, because there is only the one intrinsic cardiac regulator intact (Meyer et al 1996). This denervation result also indicates that the nervous system must produce the higher degrees of freedom found in the normal subject. Besides denervation, lowering of the heartbeat PD2i can also be achieved by blockade of the NMDA receptors, which are the synaptic mediators in virtually all of the cardiovascular centers in the brain (Skinner et al 2000).

\section{Comparison of PD2i with TWA}

Although no head-to-head comparison of PD2i and TWA has yet been made to establish their relative clinical utility, it is worth noting a comparison between them. Recently, T-wave alternans (TWA), detected by a heart-rate dependent algorithm that measures the small repolarization fluctuations of the T-wave, has shown high predictability of arrhythmic death in high-risk patients (Kop et al 2004). This method requires a stable ECG (data stationarity) of a high heart rate (treadmill) and low-noise in the ECG (special electrodes), and it is therefore difficult to apply to critically ill patients. The PD2 $\mathrm{i}$ Test has none of these limitations.

When PD2 $\mathrm{i}=1.0$, it indicates that the data have a dynamics called a limit-cycle, a dynamics which could be an alternating series of data like that the TWA device detects. At the higher heart rates TWA correlates with QT-intervals, at least in the high-risk patients (Janusek et al 2005), and thus TWA likely correlates with RR-intervals too, that is, as 
very little rate-dependent adjustments between QT and RR occur at the higher heart rates. PD2i values slightly above or below the singular value of 1.0, however, do not alternate, but rather they have a chaotic variation and look noisy, but they are not noise because they have a low dimension. It may be the case that TWA, which detects small alternating changes in repolarization, can detect the same patients where PD2i $=1.0$. But conversely, TWA could not be expected to detect the same patients at risk of VF/VT where PD2i is not equal to 1.0 .

\section{Conclusions}

We conclude that a rapid, automated, and cost-effective assessment of risk of VF/VT can be made for patient ECGs archived by NIH in a public data base. Only a 15-30 min sample of ECG data is required for an immediate assessment of the nonlinear RR-intervals by the PD2i algorithm, as embodied in the Vicor 2.0 software (ie, Ni $>1,000$ heartbeats to meet the $\mathrm{Ni}>10^{\mathrm{PD} 2 \mathrm{i}}$ criterion for $\mathrm{PD} 2 \mathrm{i}<3.0$; Kostelich and Swinney 1989). Once recorded, the data file can be examined for noise content by the PD2i acceptance ratio $(\% \mathrm{~N})$ for a priori exclusion of patients with randomized RR-intervals resulting from noise contamination, atrial fibrillation, or high arrhythmia rate ( $>10 \%$ of beats). In this way, the RR-intervals that are not suitable for nonlinear analysis can be quickly detected and excluded. The present results in public ECG data show PD2i predictability of VF/VT within the same day. These results support our recent prospective study (Skinner 2007), in which PD2i prediction of arrhythmic death was found to extend from days to weeks and months in a cohort of 400 chest-pain patients with ECG abnormalities (Skinner 2007).

\section{Disclosure}

The authors are employees and stockholders of Vicor Technologies, Inc. This work was supported in part by a grant from the National Institutes of Health, HL 069563.

\section{References}

Akselrod S, Gordon D, Ubel FA, et al. 1981. Power spectrum analysis of heart rate fluctuation: a quantitative probe of beat to beat cardiovascular control. Science, 213:220-2.

Armoundas AA, Hohnloser SH, Ikeda T. 2005. Can microvolt T-wave alternans testing reduce unnecessary defibrillator implantation? Nat Clin Pract Cardiovasc Med, 10:522-8.

Braun C, Kowallik P, Freking A. 1998. Demonstration of nonlinear components in heart rate variability of healthy persons. Am J Physiol, 275: H1577-84.

Carpeggiani C, Landisman C, Montaron M-F, et al. 1992. Cryoblockade in limbic brain (amygdala) delays or prevents ventricular fibrillation following coronary artery occlusion in psychologically stressed pigs. Circ Res, 70:600-6.
Coumel PH, Hermida JS, Wennerblöm B. 1991. Heart rate variability in myocardial hypertrophy and heart failure, and the effects of betablocking therapy: a non-spectral analysis of heart rate oscillations. Eur Heart J, 12:412-22.

Ebert PA, Vanderbeck RB, Allgood RJ, et al. 1970. Effect of chronic cardiac denervation on arrhythmias after coronary artery ligation. Cardiovasc Res, 4:141-7.

Elbert T, Ray WJ, Kowalik ZJ, et al. 1994. Chaos and Physiology. Physiol Rev, 74:1-47.

Fei L, Copie X, Malik M, et al. 1996. Short- and long-term assessment of heart rate variability for risk stratification after acute myocardial infarction. Am J Cardiol, 77:681-4.

Fleiss JL. 1981. Table A.3. Sample sizes per group for a two-tailed test on proportions. In: Statistical Methods for Rates and Proportions. Second Edition. New York: John Wiley and Sons, pp. 260-280.

Goldberger AL, Amaral LAN, Glass L, et al. 2000. PhysioBank, PhysioToolkit, and PhysioNet: components of a new research resource for complex physiologic signals. Circulation, 101:e215-e220.

Ho KK, Moody GB, Peng CK, et al. 1997. Predicting survival in heart failure case and control subjects by use of fully automated methods for deriving nonlinear and conventional indices of heart rate dynamics. Circulation, 96:842-8.

Janusek D, Karczmarewicz S, Pawlowski Z, et al. 2005. Relationship between duration of repolarization and $\mathrm{T}$ wave amplitude in patients with positive or negative T wave alternans. Kardiol Pol, 62:517-25.

Kleiger RE, Miller JP, Bigger JT, et al. 1987. The Multicenter Post-Research Group. Decreased heart rate variability and its association with increased mortality after acute myocardial infarction. Am J Cardiol, 59:256-62.

Kop WJ, Krantz DS, Nearing BD, et al. 2004. Effects of acute mental stress and exercise on T-wave alternans in patients with implantable cardioverter defibrillators and controls. Circulation, 109:1864-9.

Kostelich EJ, Swinney HL. 1989. Practical considerations in estimating dimension from time series data. Physica Scripta 40:436-41.

Marwan N, Wessel N, Meyerfeldt U, et al. 2002. Recurrence-plot-based measures of complexity and their application to heart-rate-variability data. Phys Rev E, 66:2670-2.

Meyer M, Marconi C, Ferretti, et al. 1996. Heart rate variability in the human transplanted heart: nonlinear dynamics and QT vs RR-QT alterations during exercise suggest a return of neurocardiac regulation in long-term recovery. Integ Physiol Behav Sci, 31:289-305.

Schreiber T, Schmitz A. 1996. Improved Surrogate Data for Nonlinearity Tests. Phys Rev Lett, 77:635-8.

Skinner JE. 2007. New paradigms in heart-brain medicine: nonlinear physiology and state-dependent proteomics. Clev Clinc J Med, 74: S79-S85.

Skinner JE, Beder SD, Entman ML. 1983. Psychologic stress activates phosphorylase in the heart of the conscious pig without increasing heart rate and blood pressure. Proc Nat Acad Sci, 80:4513-17.

Skinner JE, Carpeggiani C, Landisman CE, et al. 1991. The correlation dimension of heartbeat intervals is reduced in conscious pigs by myocardial ischemia. Circ Res, 68:966-76.

Skinner JE, Molnar M, Tomberg C. 1994. The point correlation dimension: performance with nonstationary surrogate data and noise. Integr Physiol Behav Sci, 29:217-34.

Skinner JE, Molnar M. 1999. Event-related dimensional reduction in the primary auditory cortex of the conscious cat are revealed by new techniques for enhancing the non-linear dimensional algorithms. Intern $J$ Psychophysiol, 34:21-35.

Skinner JE, Nester BA, Dalsey WC. 2000. Nonlinear dynamics of heart rate variability during experimental hemorrhage in ketamine-anesthetized rats. Amer J Physiol Heart Circ Physiol, 297:H1669-H1678.

Skinner JE, Pratt CM, Vybiral T. 1993. A reduction in the correlation dimension of heartbeat intervals precedes imminent ventricular fibrillation in human subjects. Amer Heart J, 125:731-43.

Skinner JE, Reed JC. 1981. Blockade of a frontocortical- brainstem pathway prevents ventricular fibrillation of the ischemic heart in pigs. Am J Physiol, 240:H156-H16. 
Skinner, JE, Verrier, RL 1982. Task Group Report: biobehavioral factors in sudden cardiac death and arrhythmias. In: Smith OA, Galosy RA, and Weiss SM (eds). Circulation, Neurobiology, and Behavior. New York: Elsevier Biomedical Press, pp. 309-16.

Skinner JE, Zebrowski JJ, Kowalik ZJ. 1998. New nonlinear algorithms for analysis of heart rate variability: low dimensional chaos predicts lethal arrhythmias. In: Kantz H, Kurths J, Mayer-Kress G (eds). Nonlinear Analysis of Physiological Data. New York: Springer, pp. 129-66.

Stein PK, Bosner MS, Kleiger RE, et al.1994. Heart rate variability: a measure of cardiac autonomic tone. Am Heart J, 127:1376-80.

Stiedl O, Meyer M. 2002. Fractal dynamics of heart beat interval fluctuations in corticotropin-releasing factor receptor subtype 2 deficient mice. Int Physiol Behav Sci, 37:311-45.
Stiedl O, Meyer M. 2003. Fractal dynamics in circadian cardiac time series of corticotropin-releasing factor receptor subtype-2 deficient mice. J Math Biol, 47:169-97.

Theiler J, Eubank S, Longtin A, et al. 1992. Testing for nonlinearity in time series: the method of surrogate data. Physica D, 58:77-94.

Vybiral T, Skinner JE.1993. The point correlation dimension of RR-intervals predicts sudden cardiac death among high-risk patients. Computers in Cardiology, 257-260.

Wagner CD, Persson PB. 1998. Chaos in the cardiovascular system: an update. Cardiovasc Res, 40:257-64. 
